\title{
The Caltech Submillimeter Observatory
}

\author{
T. G. Phillips \\ Califomia Institute of Technology, Pasadena, CA, 91106, U.S.A.
}

\begin{abstract}
A brief description of the history and technology of the Caltech Submillimeter Observatory (CSO) is presented. The design of the $10.4 \mathrm{~m}$ Leight on telescope is discussed and also the methods used for fine adjustments of the surface. The dome design is explained, plus a short description is given of the technology of the detectors.
\end{abstract}

Index Terms - submillimeter wave propagation; submillimeter wave antennas; submillimeter wave sp ectroscopy.

\section{INTRODUCTION}

The submillimeter band of the electromagnetic spectrum (wavelength $1 \mathrm{~mm}-100 \mu \mathrm{m}$ ) falls between the radio and the infrared and it is not obvious whether the techniques for telescopes and receivers will be more radio-like or infraredlike. In fact it turns out that both are needed. For high resolution spectroscopy radio techniques are used, but for low resolution or continum studies, bolometers are used. The telescopes which have pioneered the field have mostly been radio-style, using the standard design of stiff back-up structure and lightweight surface panels giving a typical rms surface error of about $20 \mu \mathrm{m}$ for a $10 \mathrm{~m}$ class telescope. From the "Ruze" formula, the shortest wavelength for good diffractionlimited performance is about $400 \mu \mathrm{m}$. Thus, for a $10 \mathrm{~m}$ telescope operating at $350 \mu \mathrm{m}$, a beam size of about 8 arc sec results. Optical telescopes produce excellent beamshapes, but with high sidelobes and at vastly greater cast. Detectors for submillimeter spectroscopy have been developed at the CSO and are now performing close to the the oretical limit given by "Quantum Noise", up to about $700 \mathrm{GHz}$. Such detectors, known as Superconductor-Insulator-Superconductor (SIS), use the phenomenon of photon assisted tunneling of quasiparticles (dressed electrons) to generate a current through a tunnel junction. They are photoconductors, but with an energy gap of the order of milli-electronvolts, matched to submillimeter energies rather than the $\sim 1 \mathrm{eV}$ of semiconductor energy gaps, suitable for the optical.

\section{HISTORY OF THE CSO}

In 1973, Dr. Robert Leighton propased to the NSF to build four millimeter-wave telescopes of $10.4 \mathrm{~m}$ diameter, three to work as an interferometer in the Owens Valley, and one as a single submm telescope on a high mountain site. The proposal was approved, except that the NSF wanted the interferometer completed before the submm project was started. Two prototypes of increasing stiffness were built before Leighton started work on what was to be the very accurate submm telescope. Figure 1 shows the CSO dish under construction at Caltech, in the large building originally constructed for work on the Palomar 200". This initial work (1975-1980) was funded in part by NSF, NASA and the Kresge foundation.

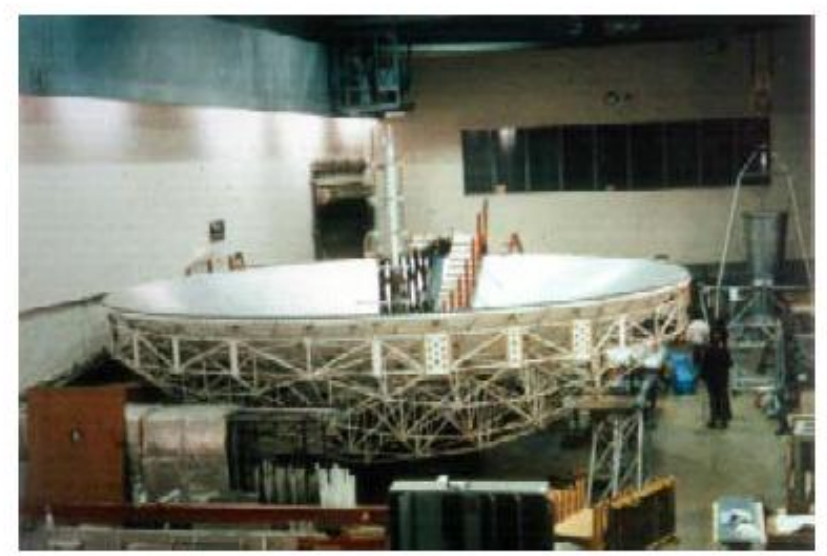

Figure 1. The submillimeter dish on an air-bearing and under the parabolic cutter-track, in the large, thermally stable building originally constructed for work on the Palomar $200^{\prime \prime}$ telescope.

At the same time as the telescope construction was starting, a decision was made to site the CSO on Mauna Kea at what is now known as Millimeter Valley - about $200^{\prime}$ below the summit - after several days of data taking by myself with a hand-held water vapor meter, to show that there was no significant difference in water column above the various possible sites. It was also necessary to file an Environmental Impact Statement which involved several in depth studies and se veral public meetings in the 1981-1983 time frame.

Again in the 1981-1983 period the dome was designed and a plan developed for a trial assembly (Figure 2) at Caltech (while the 3 element interferometer was being completed in the Owens Valley, to the satisfaction of the NSF). In 1984 the NSF approved the project and funded the construction on Mauna Kea, including the trial erection of the dome in Pasadena. The original idea of building the shutter doors on the ground and subsequently hoisting them into place on the vertical tracks (see Figure 2) proved impractical due to the wind forces. Instead the two large doors were assembled in place, on the tracks (Figure 3). The design of the shutter is that the two doors (each covering $1 / 3$ of the total dome 
segment) are actuated by a hydraulic drive and steel rope and pulley system, the front door moving at twice the rate of the upper door, so that the two nest together at the back when fully open. In spite of the trial erection, the construction company suffered great difficulty on the mountain and eventually went bankrupt, leaving the completion of the Mauna Kea construction to the Caltech staff. Nevertheless, the job was completed in time for the dedication in November, 1986. The completed observatory is seen in Figure 8.

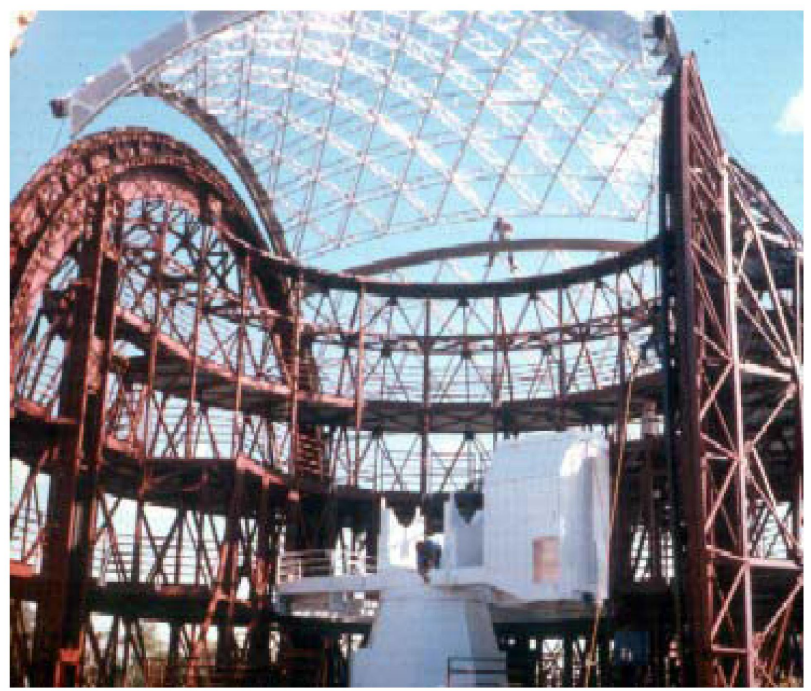

Figure 2. The trial erection of the dome at Caltech. The technique of hoisting part fabricated door frames onto the track, using a crane, proved too susceptible to wind forces even in the light breezes of Pasadena.

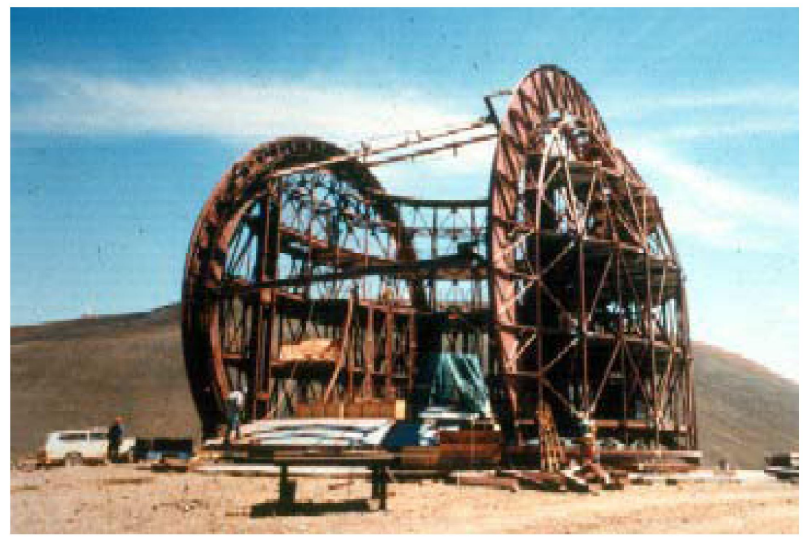

Figure 3. Assembly of the dome on Mauna Kea. Here the shutter doors are being assembled on the tracks "in situ".

\section{NOVEL TECHNOLOGY ASPECTS}

The CSO incorporates many innovative technologies in the telescope, dome, receivers and bolometers. The radio style dish [1] has a stiff, but homologous steel tube back-up structure, with only pinned joints so there are no shear forces, to enable accurate modeling. The panels are light-weight aluminum honeycomb hexagons (the hexagon concept was later followed by the Keck telescopes). Leighton's construction technique was to mount the structure on an airbearing, to rotate under a parabolic track set to an accuracy of about $2 \mu \mathrm{m}$ by laser ranging, relative to an oil tray as a gravitational level. A cutter runs on the parabolic track to machine the upper honeycomb surface (Figure 4). Thin aluminum sheets are glued and sucked down onto the machined surface of each honeycomb hexagon, later to be ground and polished to final shape. In this way Leighton achieved a primary surface accuracy of about $10 \mu \mathrm{m}$.

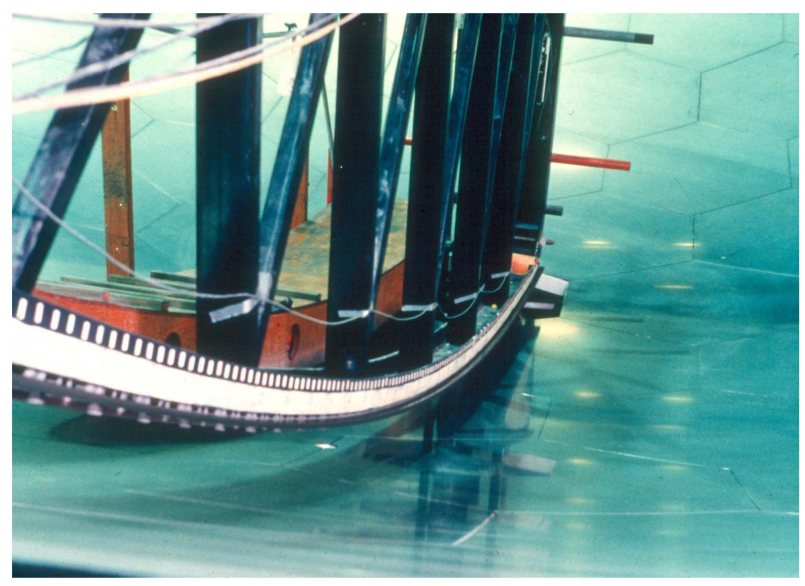

Figure 4. The parabolic cutter track was also used for grinding and polishing the surface. A final accuracy $(1 \sigma)$ of $10 \mu \mathrm{m}$ was achieved in the lab.

The primary structure was broken into three for shipping to Hawaii and when reassembled at the site was found to have a surface error of about $40 \mu \mathrm{m}$. This was reduced to about 20 $\mu \mathrm{m}$ by various procedures.

Three independent techniques were planned to enable correction of the primary mirror. The panels are supported from the back-up structure by steel stand-off pins. The first and coarsest method is to adjust by hand a differential screw at the bottom of each stand-off pin. A more refined method to adjust the length of the pin is with a Peltier (heater/cooler) unit [2]. This was not implemented until recently (Figure 5). The third correction is to the curvature of each panel by means of a warping harness. This is needed because the dish was originally set in an ambient temperature room, but operation is with the surface exposed to the cold night sky, causing a flow of heat from the rear of each panel to the front causing a change in the curvature, in spite of styrofoam insulation on the back faces and edges of the panels.

All of these correction techniques require some measurement of the surface errors. At the CSO we use a novel scheme which is a single dish analog of a two element 
interferometer holographic system [3]. One beam looks at a planet as a phase reference, the other makes a far-field map of the beam in the direction of the planet and the two are multiplied. In the case of the CSO the second beam is obtained with a beam splitter and a rotating mirror (two dimensions). The resulting far-field beam maps are transformed to give a phase map in the aperture plane which provides the required corrections. The scheme provides error correction maps as a function of elevation angle to about $7 \mu \mathrm{m}$ accuracy. The heating/cooling of the stand-off pins generally improves the efficiency by about $50 \%$ at the highest frequencies.

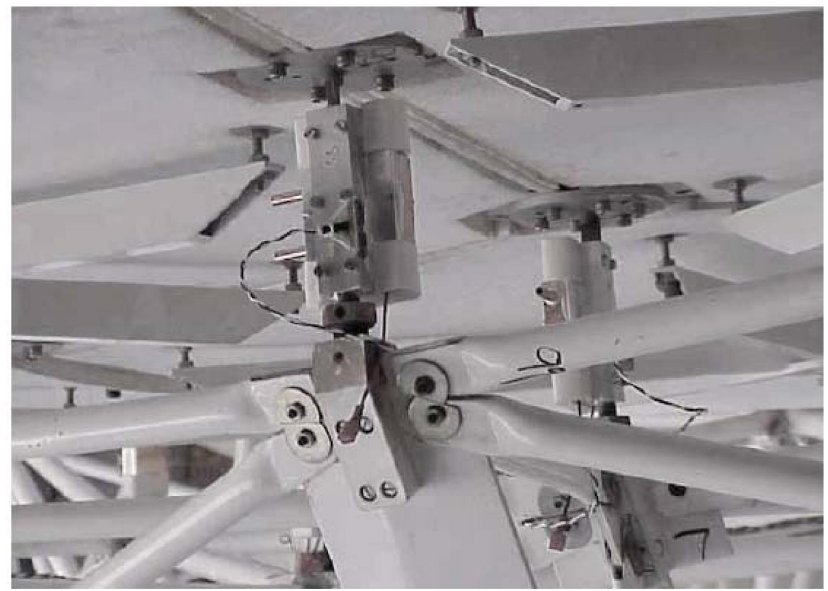

Figure 5. A view of one of the Peltier units which control the lengths of the stand-off pins. There are 88 such units on the telescope.

The CSO has a full set of heterodyne receivers, covering all atmospherically allowed frequencies in the $180-950 \mathrm{GHz}$ band. They are all SIS receivers, [4] (which is the style of receiver chosen for most of the bands for ALMA). While the IF bandwidth is limited to $1 \mathrm{GHz}$ for most receivers currently, we are rebuilding them all to have the IF range $4-8 \mathrm{GHz}$. Such a receiver is shown in Figure 6, where there is a balanced input which is useful for eliminating local oscillator noise. The dual detector system can also be used as dual polarization or sideband separating or on-off switching style receivers. The receiver noise temperatures increase roughly linearly with frequency, at about three times the theoretically limiting "Quantum Noise" up to the band gap frequency of niobium at $\sim 700 \mathrm{GHz}$.

The CSO also supports bolometer cameras; Bolocam (141 pixels at 1 and $2 \mathrm{~mm}$ ) and SHARC II (384 pixels at 450 and $350 \mu \mathrm{m})$.

Other devices supported include a Fabry-Perot, a grating spectrometer, a polarimeter and a Fourier transform spectrometer.

For the future there is a development of a new superconducting device - a Kinetic Inductance Detector - which will replace bolometers in many applications. They can function as multicolor pixels with multiplexed output, providing cameras with thousands or tens of thousands of pixels [5].

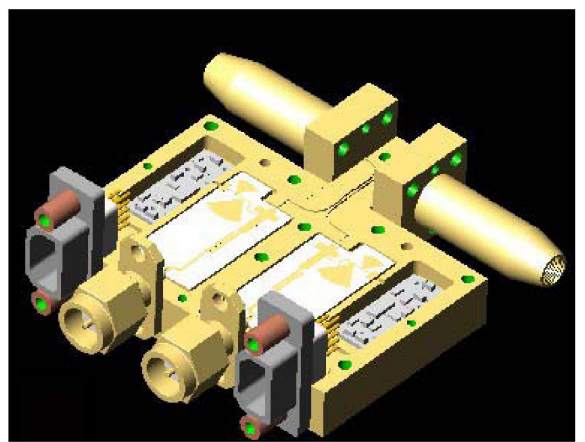

Figure 6. A view of a modern SIS receiver block with the top removed. The two feed horns provide signal and local oscillator input and a balanced front-end.

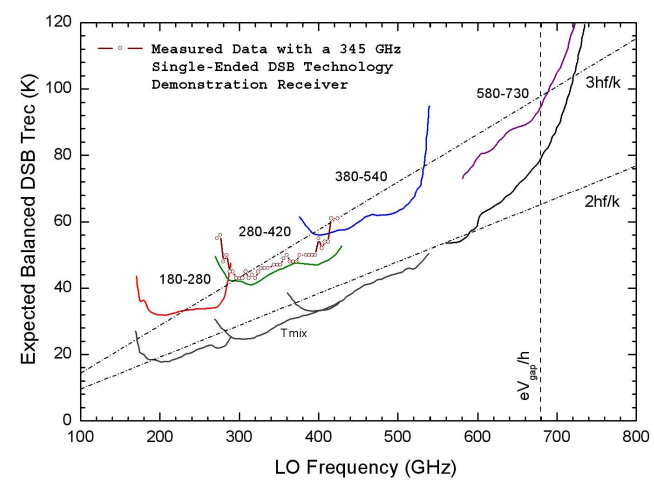

Figure 7. CSO receiver noise temperatures as a function of frequency.

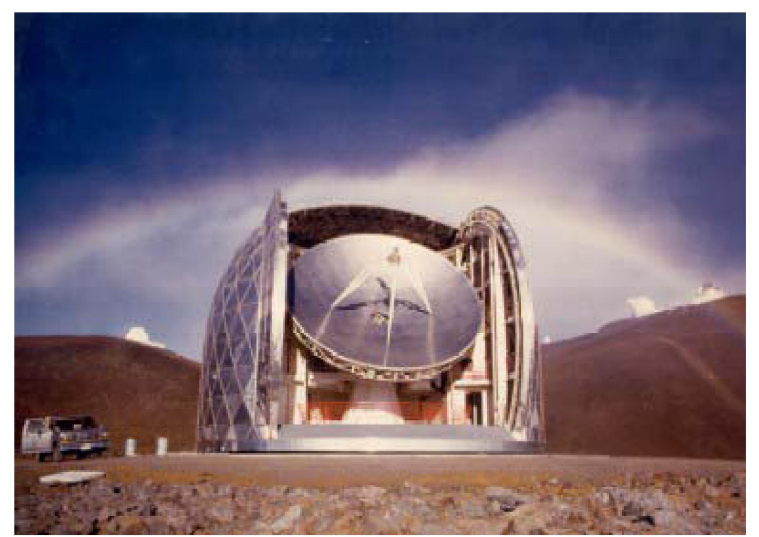

Figure 8 . The completed observatory. The dome azimuth drive is slaved to follow the telescope which allows internal room for control and receiver and detector functions. At any given time the CSO supports 5 heterodyne receivers (Cass and left Naysmyth), 2 cameras (Bolocam - Cass), (SHARC II-right Naysmyth), and the grating-(right Naysmyth). 


\section{ACKNOWLEDGEMENT}

The CSO is funded from NSF contract \#AST-0540882.

\section{REFERENCES}

[1] Robert B. Leighton "A 10 Meter Telescope for Millimeter and Submillimeter Astronomy" Final Technical Report for NSF Grant 73-04908, 1978.

[2] M. Leong, R. Peng, M. Martin, H. Hiroshige, R. Chamberlin, T.G. Phillips "A CSO submillimeter active optics system" in Proceedings of the SPIE, Volume 6275, 2006, 21L.

[3] E. Serabyn, T.G. Phillips, C.R. Masson "Radio Telescope Surface Measurement with a Shearing Interferometer" in URSI Proceedings, Holography Testing of Large Radio Telescopes, 1991, 40.

[4] T.G. Phillips \& D.P. Woody "Millimeter and SubmillimeterWave Receivers” An. Rev. Astron. Ap, 1982, 20, 285.

[5] P. Day, H.G. LeDuc, B. Mazin, A. Vayonakis, J. Zmuidzinas "A superconducting detector with a multiplexable microwave readout" Nature, 425, 2003, 817. 